\title{
Coping with medical error: a systematic review of papers to assess the effects of involvement in medical errors on healthcare professionals' psychological well-being
}

\author{
Reema Sirriyeh, ${ }^{1}$ Rebecca Lawton, ${ }^{1}$ Peter Gardner, ${ }^{1}$ Gerry Armitage ${ }^{2}$
}

\begin{abstract}
- Supplementary appendices are published online only. To view these files please visit the journal online (http://qshc.bmj. com).

${ }^{1}$ Institute of Psychological Sciences, University of Leeds, Leeds, UK

${ }^{2}$ Bradford Institute for Health Research, Bradford, UK
\end{abstract}

\section{Correspondence to} Reema Sirriyeh, Institute of Psychological Sciences, University of Leeds, Leeds LS2 9JT, UK;

r.h.sirriyeh07@leeds.ac.uk

Accepted 7 January 2010 Published Online First 31 May 2010

\begin{abstract}
Background Previous research has established health professionals as secondary victims of medical error, with the identification of a range of emotional and

psychological repercussions that may occur as a result of involvement in error. ${ }^{23}$ Due to the vast range of emotional and psychological outcomes, research to date has been inconsistent in the variables measured and tools used. Therefore, differing conclusions have been drawn as to the nature of the impact of error on professionals and the subsequent repercussions for their team, patients and healthcare institution. A systematic review was conducted.
\end{abstract}

Methods Data sources were identified using database searches, with additional reference and hand searching. Eligibility criteria were applied to all studies identified, resulting in a total of 24 included studies. Quality assessment was conducted with the included studies using a tool that was developed as part of this research, but due to the limited number and diverse nature of studies, no exclusions were made on this basis.

Results Review findings suggest that there is consistent evidence for the widespread impact of medical error on health professionals. Psychological repercussions may include negative states such as shame, self-doubt, anxiety and guilt. Despite much attention devoted to the assessment of negative outcomes, the potential for positive outcomes resulting from error also became apparent, with increased assertiveness, confidence and improved colleague relationships reported.

Conclusion It is evident that involvement in a medical error can elicit a significant psychological response from the health professional involved. However, a lack of literature around coping and support, coupled with inconsistencies and weaknesses in methodology, may need be addressed in future work.

\section{INTRODUCTION}

Involvement in errors has been found to elicit a significant emotional response from health professionals. ${ }^{1}$ While the impact of error on patients is well documented, there are only two reviews that discuss the impact of medical error on professionals. $^{2}{ }^{3}$ Both fail to provide an adequate discussion of findings relating to coping or outcomes beyond the immediate error event. There remains a need to identify factors that moderate the emotional effects of an error on the individual, and to understand how individuals cope with being involved in an error in the short- and longer term. It may then be possible to develop effective support mechanisms that serve the needs of different people and reduce the emotional burden associated with making an error. Therefore, the research questions posed for this review were:

1. What is the impact of being involved in a medical error on the health professional?

2. How do health professionals cope in the shortand longer term when they have been involved in a medical error?

3. Are there any factors (referred to below as moderating factors) that influence the immediate response to error and/or the way in which individuals cope?

\section{METHODS}

\section{Search identification and selection}

Electronic databases (Web of Science, Medline 1950-2009, PsychInfo 1967-2009, Science Direct, The Cochrane Library, Embase 1980-2009) were searched using terms that covered both attitudes towards making errors, as well as the responses following such errors. Seven distinct searches were conducted to retrieve all of the relevant information as displayed in table 1. An information scientist checked the search strategy to ensure the capture of all relevant articles. All searches were limited to studies on humans, written in the English language and published between 1980 and 2009. The reference lists of all relevant articles identified were handsearched.

The Web of Science returned 16 of the included studies with a further four studies from the remaining databases (Medline/Pubmed, PsychInfo 1980-2009, Science Direct, The Cochrane Library, Embase). Reference list searches identified all additional articles.

\section{Eligibility criteria}

Inclusion criteria

- types of error: the error studied must fit the definition:

the failure of a planned action to be completed as intended or the use of a wrong plan to achieve an aim without the intervention of an unforeseen event' (Reason 1990)

- types of participants: given the core aims of the review, all studies were required to include health professionals and trainees from any specialities as participants in at least part of the sample.

- research aims: studies must focus largely on the psychological or emotional outcomes for health professionals in the event of an error. 
Table 1 Search terms

\begin{tabular}{ll}
\hline $\begin{array}{l}\text { Search } \\
\text { no }\end{array}$ & Search history \\
\hline 1 & $\begin{array}{l}\text { coping with medical (error or mistake or mishap or blunder or adverse event } \\
\text { or incident) } \\
\text { respond to medical (error or mistake or mishap or blunder or adverse event } \\
\text { or incident) }\end{array}$ \\
3 & $\begin{array}{l}\text { attitude towards medical (error or mistake or mishap or blunder or adverse } \\
\text { event or incident) } \\
\text { commit medical (error or mistake or mishap or blunder or adverse event or } \\
\text { incident) }\end{array}$ \\
6 & $\begin{array}{l}\text { perception of committing medical (error or mistake or mishap or blunder or } \\
\text { adverse event or incident) } \\
\text { making medical (error or mistake or mishap or blunder or adverse event or } \\
\text { incident) } \\
\text { recover from making medical (error or mistake or mishap or blunder or } \\
\text { adverse event or incident) }\end{array}$ \\
\hline
\end{tabular}

- study design: studies must employ a quantitative, qualitative or mixed design.

- settings: studies must take place in primary, secondary, tertiary and community care settings; this does not include alternative/complementary therapy settings or dentistry.

Articles were excluded if they were not:

- empirical studies and original studies;

- published in English;

- published in peer-reviewed journals;

- published between 1980 and 2009; prior to 1980, this issue was not explicitly acknowledged, and so may be difficult to depict objectively from papers.

\section{Method of review and data extraction}

Results were merged using reference-management software (Endnote), and duplicates removed. All articles were checked by one reviewer [RS] against the inclusion and exclusion criteria. Full texts of the retained papers were then obtained, and these papers were then assessed against the eligibility criteria. The following data were then extracted from each paper: investigator(s), publication date, study design, outcome measures, key findings and findings related to the emotional response and coping after involvement in a medical error. The extracted articles ${ }^{4}$ were then checked by the second and third reviewers (RL, GA) against the inclusion and exclusion criteria. The inclusion of 20 articles, with a further four articles from the hand search, was agreed. Figure 1 shows reasons for exclusion.

The heterogeneous measures and research questions excluded the use of a meta-analysis of this literature. Therefore, the threestage UK Evidence for Policy and Practice Information and Coordinating Centre (EPPI) approach for synthesis of diverse studies was not considered appropriate here. ${ }^{5}$ Findings were synthesised across all the papers in a narrative empirical synthesis; an alternative EPPI approach which has been used in other similar reviews. ${ }^{6}$ Data that addressed our specific research questions were extracted from each paper to create themes and categories.

\section{Assessment of study quality}

Assessing the quality of evidence from both qualitative and quantitative studies was challenging. Based on earlier approaches, including criteria developed by EPPI-centre, a set of 17 quality assessment criteria had been developed (see appendix 1). ${ }^{7-10}$ However, initial attempts by different members of the author team to apply these criteria highlighted some difficulties.

These included the difficulty in assessing criteria on a dichotomous scale (yes/no), variability in the interpretation of the criteria by reviewers, omission of key quality issues in methodology and analytical strategy, and suitability of wording in relation to qualitative work. Dichotomous responses failed to distinguish between studies with strong and weak coverage of an issue which affected inter-rater reliability-for example, assessment of valid and reliable outcomes could incorporate sophisticated statistical assessment or merely face-validity checks. For some criteria, for example 'Clear description of sample,' key quality issues, such as the adequacy of the sample size, were omitted. Finally, the reliability of the analytical process in qualitative work was not considered adequately, with
Figure 1 Flow diagram of search and retrieval process.

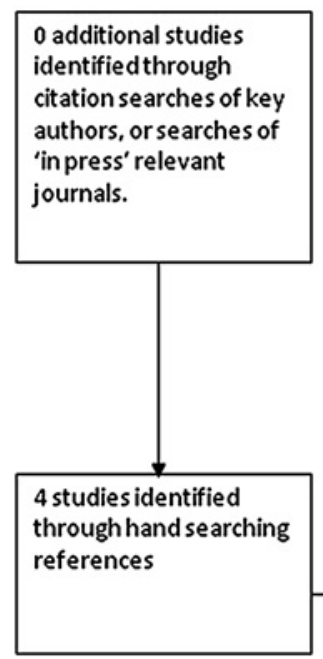

1798 exclusions:

Potentially relevant studies identified through initial searches ( $n=1823$ )

76 did not meet date criteria

194 were not published in English

1460 were not considered to be topic relevant from the title

66 were not empirical studies

Studies retrieved formore detailed evaluation $(n=25)$

2 were not in peerreviewedjournals

6 exclusions:

2 did not focus on pertinent issues

Met inclusion criteria \& included in review $(n=19)$

4 did not meet participant criteria 
only the validity of results mentioned in the list of criteria. Following consultation with experts in the field (eg, York Centre for Reviews and Dissemination) we were unable to identify a more sophisticated quality assessment measure, which led to the adaptation of the original 17 criteria in the EPPI tool for use in this review.

Studies were therefore assessed against the adapted criteria, each scored on a four-point scale. To reduce the need for expert raters, the requirements for each score were clearly defined (see appendix 2). Using this method, each paper was given a quality score, and an overall score for the body of evidence was produced. One reviewer (RS) assessed all studies against the criteria, and there was substantial agreement $(\kappa=67.8 \%)$ between reviewers (RS, RL, PG) on a random sample of papers. ${ }^{5} 11$

Studies were consistently rated highly on descriptions of methods yet generally received low scores on criteria related to study design and reasons for the choice of measure(s). Without a clear theoretical basis, and in the absence of an obvious choice of measurement, it appeared that measures were often selected with no clear justification. The fact that the adequacy of sample size was not considered raised questions about the power of some studies. Participants were rarely involved in study design; the changes made as a result of pilot work were not made in respect of participant views (see appendix 3 for a comparison table of the different types of study).

\section{RESULTS}

The electronic search yielded 1925 studies, and following the process described above, 24 studies were included in the review (see figure 1). The key characteristics of the reviewed papers are displayed in table 2 .

\section{Key findings}

\section{Response and impact}

Addressing research question 1, a state of significant emotional distress, for example, shame, guilt, fear, panic, shock and humiliation, immediately following an error was consistently raised in all papers. Broader psychological responses, such as self-doubt, loss of confidence and altered perspectives of patient and colleague relationships, were also frequently reported. ${ }^{13-17}$ The impact of making an error was widespread. Common states of emotional distress, including anxiety, depression and guilt, often transferred into personal life. ${ }^{13-15}{ }^{18-21}$ In the workplace, negative outcomes included the loss of professional reputation, distrust and reduced goodwill towards patients, and detachment from patients. ${ }^{15} 1622$ Some authors described positive outcomes in practice resulting from an error, although often somewhat briefly. For example, improvements in practice occurred as a result of error including personal practice changes, corrective actions within the department and hospital-wide actions, often relating to increased communication with other departments. ${ }^{17}{ }^{23}$ It was reported that $70 \%$ of their sample of physicians described improved professional relationships following discussion of an error, and that improved communication following an error was due to increased assertiveness in the workplace. ${ }^{24} 25$ It is evident that the psychological response to error could be mediated by the error outcome, subsequent patient relations, team response and institutional handling of the error. Greater intensity of emotional distress resulted from poorer patient outcomes, with higher selfblame also found in these cases. ${ }^{15} 1926$ A linear relationship was also described between error severity and intensity of emotional outcome for the professional, and increased emotional burden when staff considered the institutional handling of error to be poor. ${ }^{4}{ }^{17}$ Reactions of managers and senior staff were described as inconsistent, and even disapproving or aggressive, with staff experiencing interrogation, blame or occasional threats. ${ }^{14} 27$ However, retaining good patient relationships or good support from colleagues following an error had a positive effect, increasing the likelihood of a good emotional outcome for the health professional and increasing their confidence. ${ }^{15} 16$ This pattern of response is reflected in the first three stages of a six-stage response and recovery trajectory from the moment the error is realised to the influence it has in ongoing working life. ${ }^{17}$

\section{Coping and learning}

The latter three stages of the recovery trajectory proposed by Scott et al advance to the disclosure and the professional implications, support seeking and outcomes for an individual's longerterm healthcare career. ${ }^{17}$ This review revealed that many more studies have explored the attitudes and immediate responses to error, $^{24}$ rather than the management, coping or longer-term outcomes of error beyond brief references to these issues, ${ }^{8}$ but the findings identified around coping reflected these three areas.

The limited evidence relating to research question two suggests that there was a lack of support for the individual following an error, and limited self and organisational learning. Emotional support was often sought from close friends and family, but discussing details of the event with colleagues was found to be invaluable. ${ }^{17} 1928$ Despite this, support in the workplace was reported to be insufficient and, at times, even detrimental. ${ }^{13} 142729$ Error outcome was identified as a possible moderator of the emotional response and the type of coping following an error. Muller and Ornstein demonstrated a positive relationship between the severity of the error outcome and the emotional turmoil that followed, suggesting that coping was more difficult after a poor outcome. ${ }^{4}$ Conversely, Crigger and Meek reported better coping where an adverse event had occurred that was accepted, disclosed and resolved. ${ }^{30}$ They suggested that an unreported near-miss scenario may be potentially more damaging because the individual might rationalise the event internally, causing distress in the longer term, as the issue is never resolved. Despite limited discussion of near-miss data, this information could be invaluable in informing a strategy for learning. Using the experience of an error constructively for learning within teams and to aid coping was a recurring finding. ${ }^{16} 172429$ Kroll et al identified constructive feedback in the formal discussion of error as a key component in the effective use of error for learning. ${ }^{16}$ Scott et al make an important distinction between 'surviving' and 'thriving' in recovery from an error event. Individuals may function at the expected performance level but continue to be disturbed by the error event, where others may experience enhanced performance as a result of actively engaging in improving practice after a negative clinical incident. ${ }^{17}$ The importance of handling error in the correct way with a trainee sample was also highlighted, that is, avoiding punitive action or negative responses. ${ }^{21} 31$ Wolf et al suggested that the inadequate management of error resulted in less learning, and poorer coping going forward. ${ }^{27}$

\section{Attitudes in the context of culture}

Attitudes towards error are important for error management at different levels of the organisation and are developed and maintained by the healthcare culture. Several papers therefore explicitly studied the socialisation of those in medical training. ${ }^{9}$ It was suggested that the ideology of internal accountability and infallibility resulted in profound self-doubt and guilt in medical trainees. ${ }^{18} 31$ Similarly, other key factors that influenced attitudes towards error were highlighted in qualitative work and 

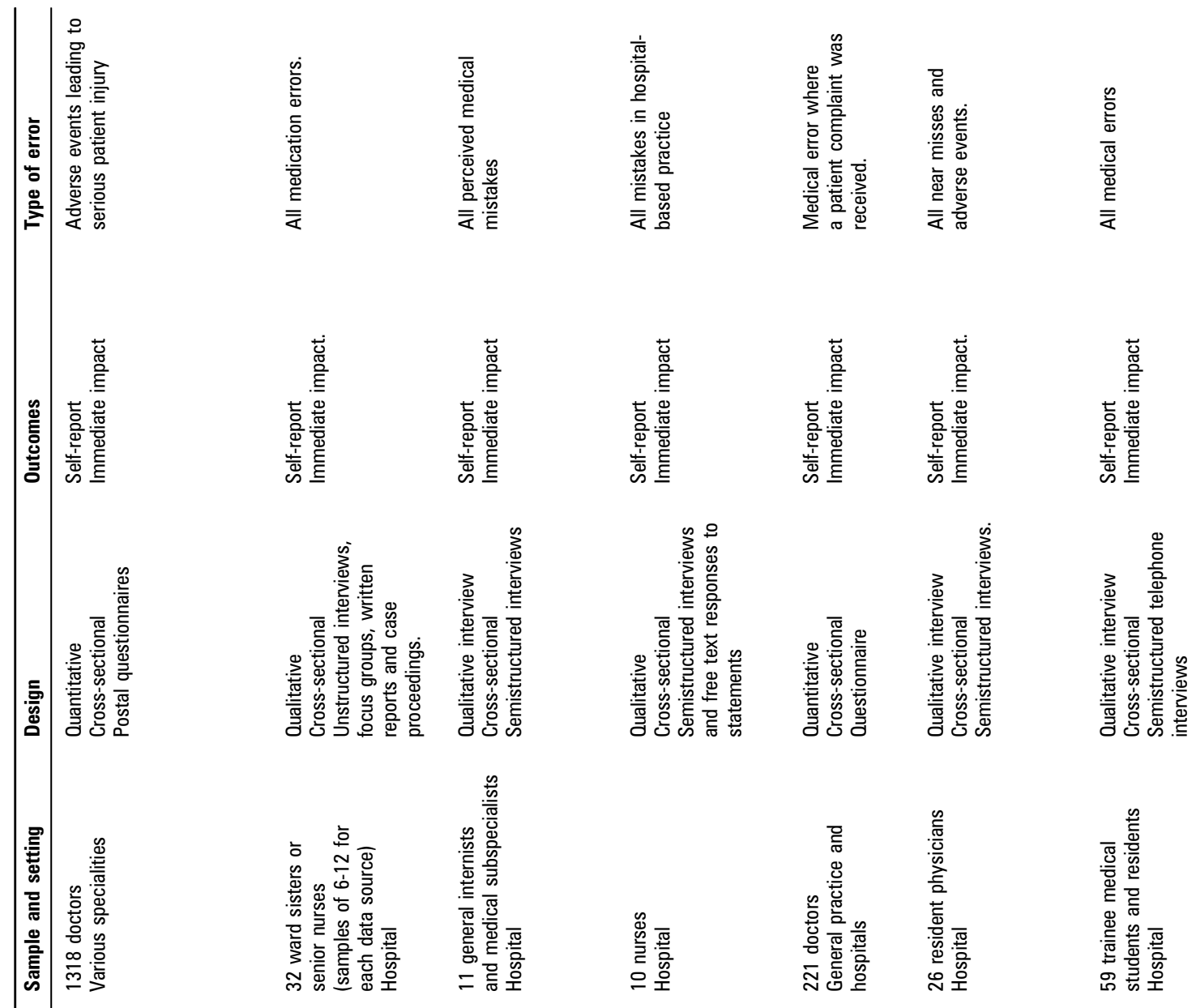

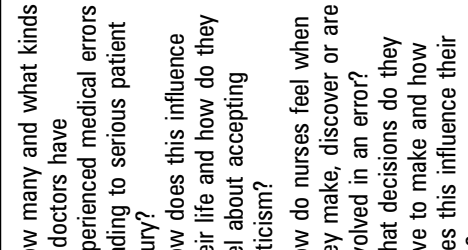
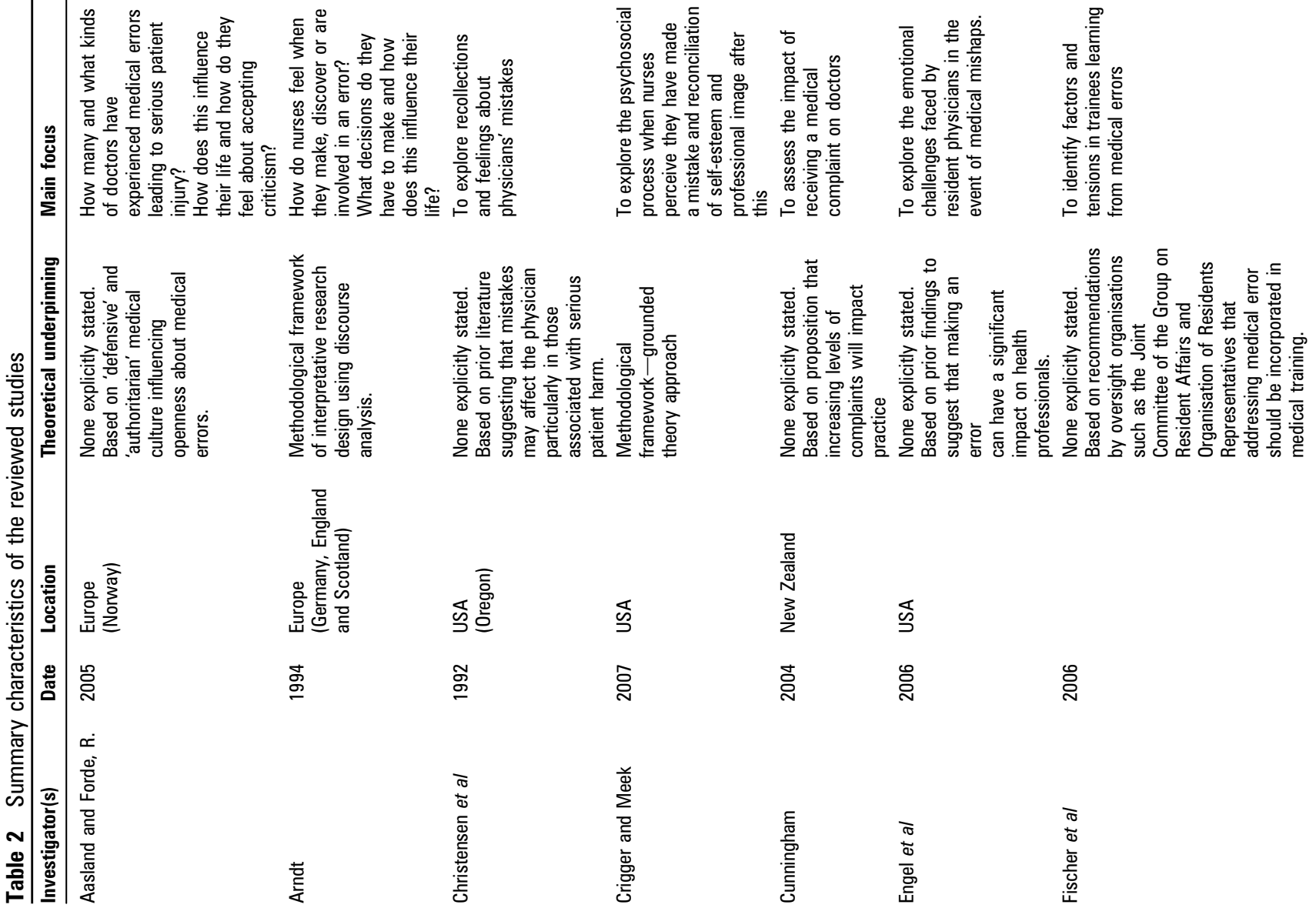

嵩密

奇

葟
ฮั

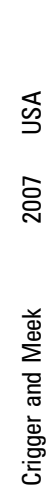

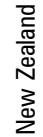

高

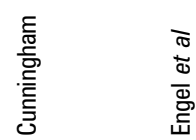




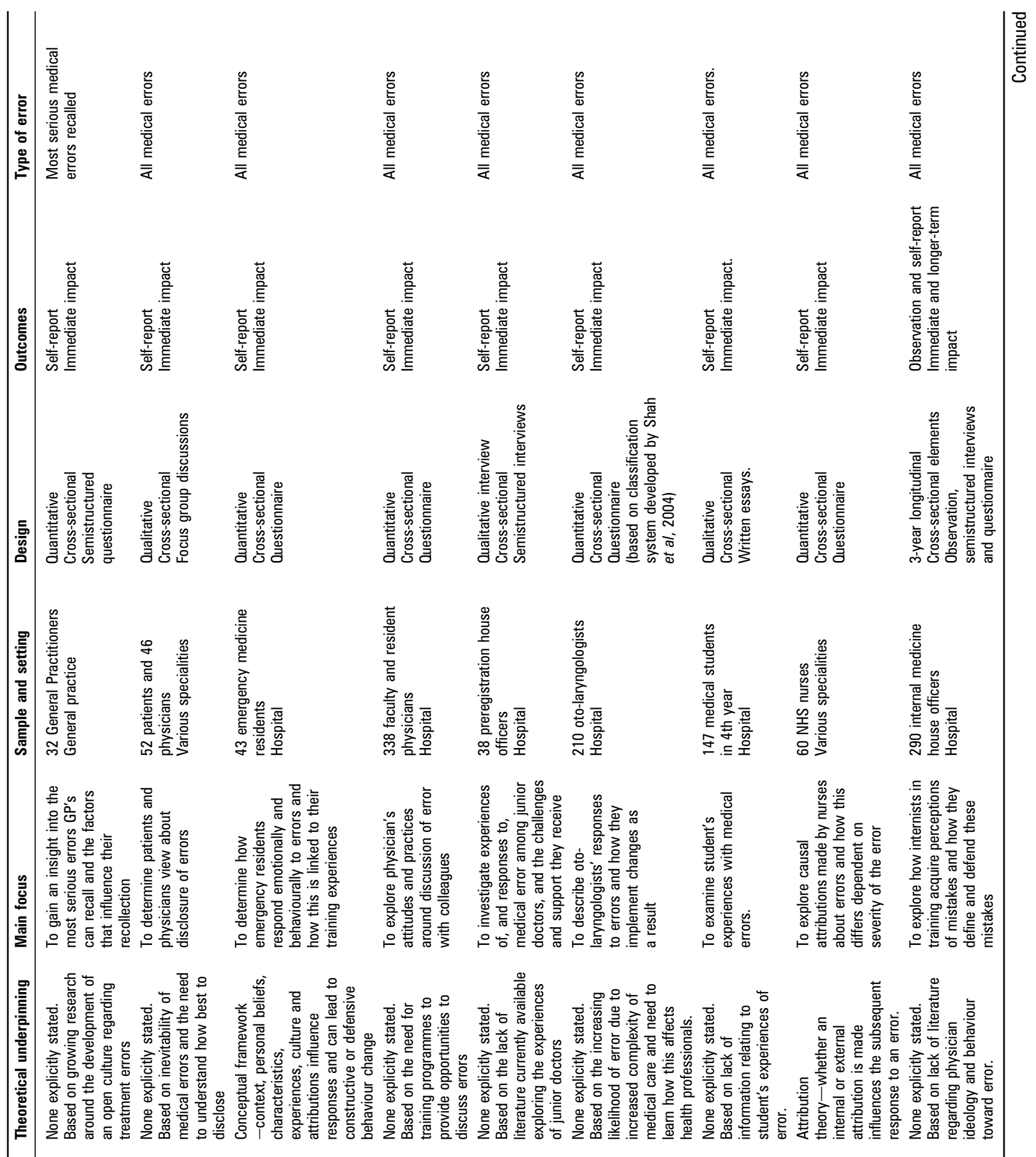

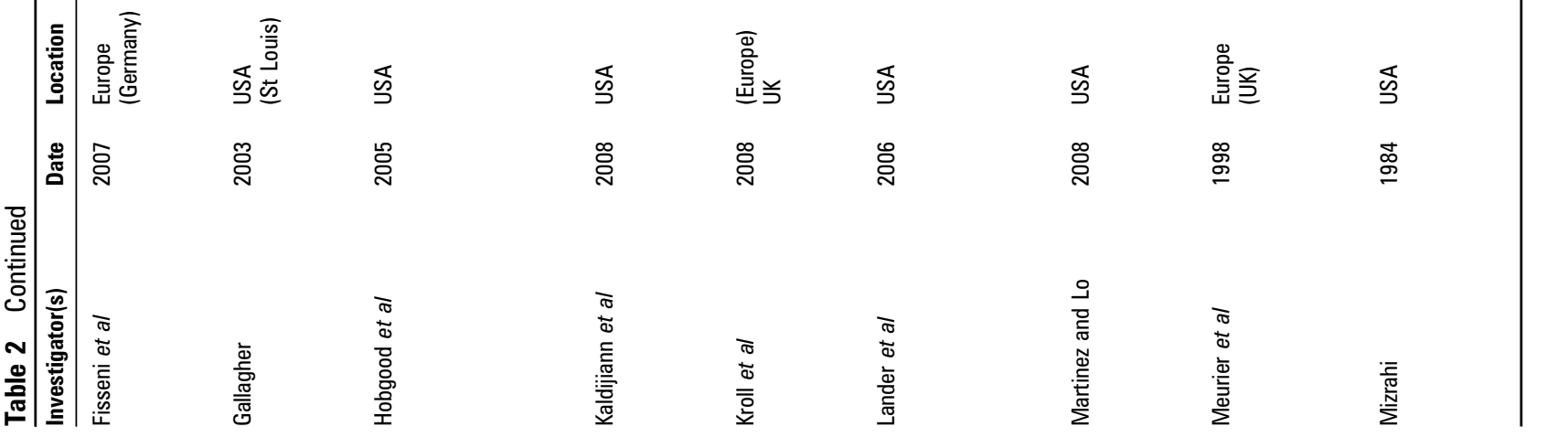




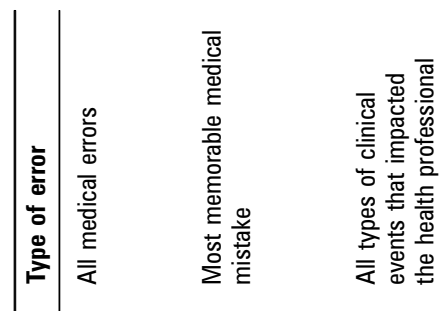

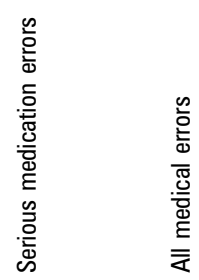

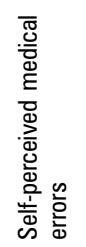

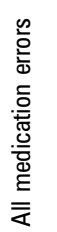

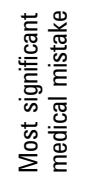

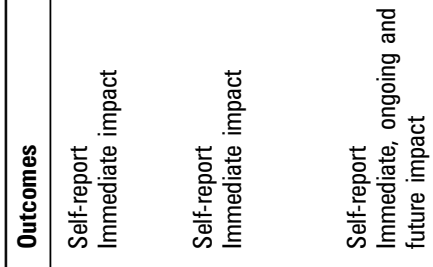

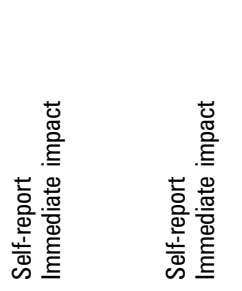

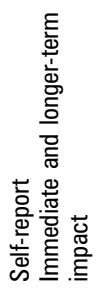

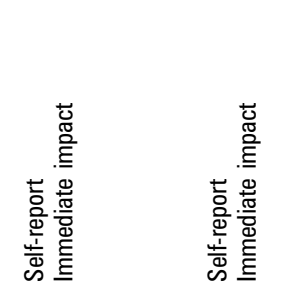

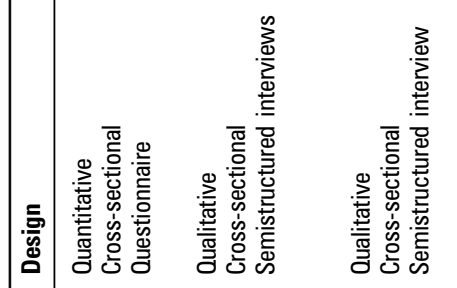

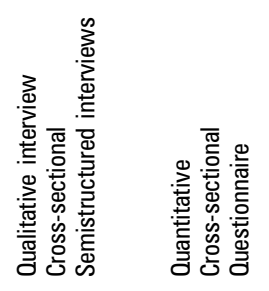

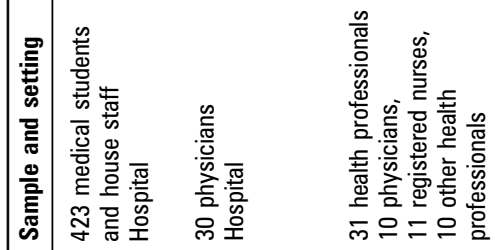
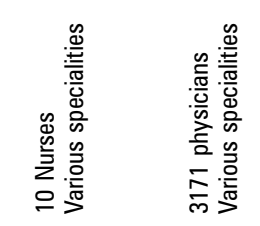

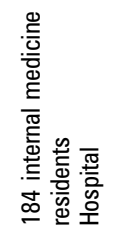

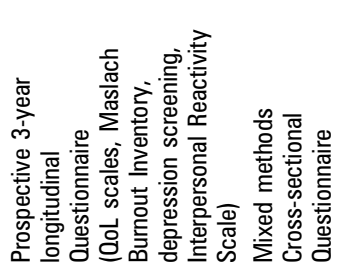

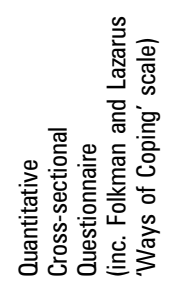

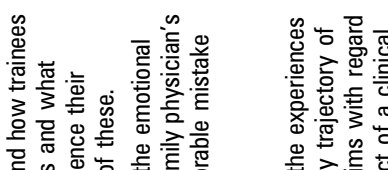

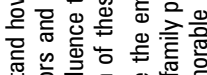

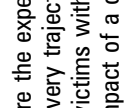

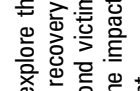

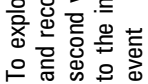

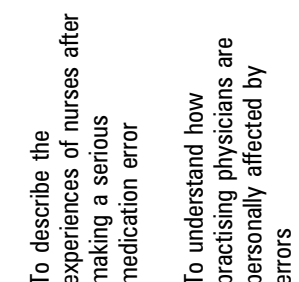

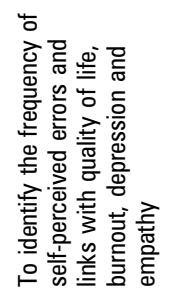

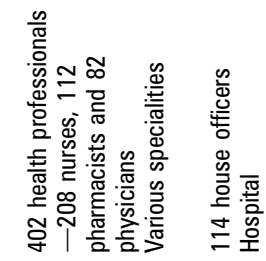

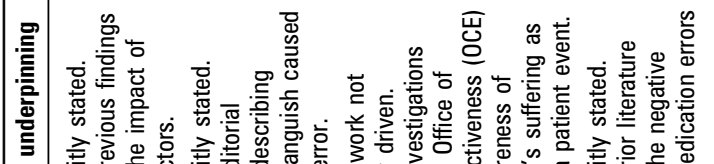

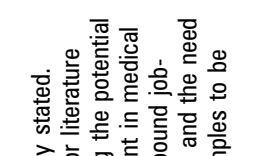

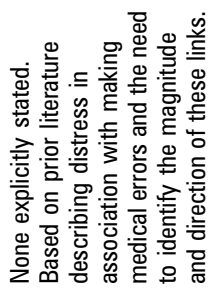

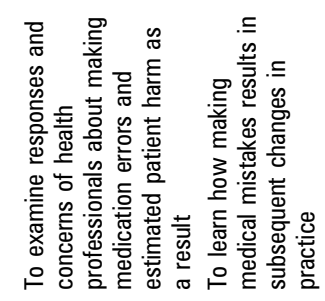

ब्ञ

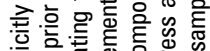

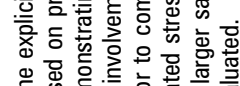

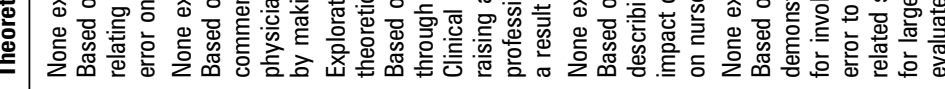

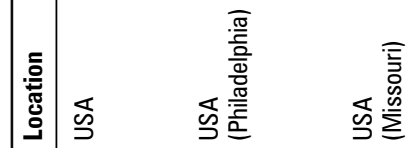

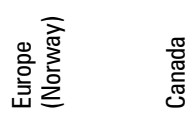

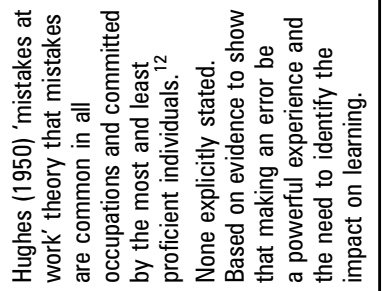

ฐัฐ

옹

ఫิ పे

药

高

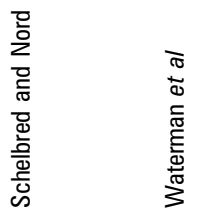

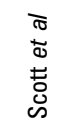

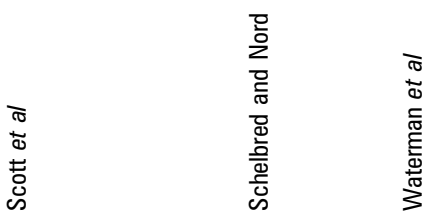

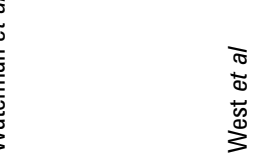

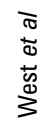

$\frac{\sqrt{3}}{\frac{10}{0}}$ 
included the hierarchical influence within the workplace, the threat of litigation and experiences of error. Lower-grade workers experienced disapproving responses to error disclosures by managers and were discouraged from disclosing errors by senior colleagues. ${ }^{27} 31$ Such negative attitudes appeared to be linked to the fear of litigation and professional loyalty. Professionals indicated that discussing error was unsafe, as it may lead to punitive action against those involved, which may damage relationships with colleagues. ${ }^{15} 173233$ Such fear was highlighted as a key barrier to the discussion and reporting of error. Conversely, Waterman et al reported that $90 \%$ of physicians experienced inadequate support with colleagues 'turning a blind eye' following an error. ${ }^{13}$ However, interestingly, good colleague support was found to be valuable in recovery from an error. ${ }^{32}$ Despite the lack of studies that explicitly address factors that influence the coping of staff following an error, the research described here suggests that attitudes, culture and colleague relationships may be important.

\section{Moderating factors and implications for practice}

The extent to which the response to error varies as a function of clinical setting and professional group is not clear. A disproportionate amount of research is conducted within hospital settings ${ }^{16}$ compared with primary care. ${ }^{1}$ Despite the remainder of studies ${ }^{8}$ drawing together a sample from across these two care environments, comparisons were not made between these two respondent groups. This has implications for the management of error in primary care settings when so little is known about the experiences of staff in these settings. A greater proportion of studies focused on either trainee or experienced physician samples ${ }^{19}$ in comparison with nurses, ${ }^{5}$ but differences in the nature of the research and responses were evident between these professional groups, such that research with nurses was predominantly qualitative with research questions directed at the discussion of feelings, emotions and the meaning of making an error to these individuals. Nurses were reflective in their response to making an error, with these events having an influence over the way they felt about themselves and how they work. $^{26} 30$ Nursing samples were also consistent in raising a feeling of personal responsibility for an error and the commitment to reporting such incidents regardless of an increased likelihood of being blamed. ${ }^{14} 242630$ Papers featuring trainee samples highlighted the learning element of making an error, focussing on clinical outcomes and change in practice. ${ }^{16} 193134$ All studies that explored only adverse events, or the most memorable or significant error, included samples limited to physicians, resulting in increased focus on the activities taken to manage the clinical outcomes of the error and professional repercussions of these events. ${ }^{15} 22293235$ Comparisons of the emotional responses of these different professional groups are hampered by the different research questions posed for the two group. This also has implications for attempts to use current knowledge about the impact of error to inform change.

\section{DISCUSSION}

This is the first review to take a systematic and structured approach to identifying the current state of research into the attitudes, responses and coping strategies of health professionals who make errors. Twenty-three studies, across a number of healthcare settings, were reviewed.

\section{Key findings}

Little is known about the proportion of individuals who suffer as a consequence of making an error, or the relationship between severity of error and the experience of distress. Nevertheless, the literature suggests that following an error feelings of shame, guilt, anxiety, fear, self-doubt and depression are consistently reported. Making an error has implications for professional and personal life, threatening reputation, self-confidence, and relationships with colleagues and patients. ${ }^{13-15} 1735$ Medical culture drives the development and maintenance of attitudes that influence responses to and management of error, such that a lack of formal support and poor institutional handling of errors are frequently reported. $^{4} 16 \quad 182731$ Interestingly, although peer support is important for both individual and team recovery, willingness to provide such support to colleagues following an error is lacking. ${ }^{13}$ The six-stage recovery trajectory proposed by Scott et al reflected many of the key findings from this review and invites further investigation on a larger scale towards a basis for the development of institutional support. ${ }^{17}$

Despite the developing body of research, many issues are yet to be addressed. Gaps in knowledge currently exist around coping, formal and informal support systems and longer-term outcomes for the professional. Greater discussion of successful coping could be highly informative for intervention design. To date, the potential moderators of the emotional response, which may include error severity, colleague relationships, workplace culture and individual characteristics such as personality, have not been studied explicitly. These factors could be central in understanding the circumstances in which support would be beneficial, and may alert both individuals and healthcare institutions of the circumstances in which an error may have the most severe repercussions. Although the negative impact of an error has been well documented, there is little consideration of the possible positive affective outcomes that may allow a more constructive use of error for learning and improvement.

\section{Methodological limitations of reviewed papers}

The wide range of measurement tools found in the review may be advantageous in one sense, allowing for a broad range of outcomes to be assessed, but at the same time this makes comparisons across studies difficult. For instance, no two quantitative studies use the same instrument to assess attitudes or emotional response. Similarly, there is an absence of commonly used general health and specific mental health measures such as the General Health Questionnaire or Beck Depression Inventory. ${ }^{36} 37$

A large number of studies rely on survey methods which may not be sufficient to study such potentially intense emotional reactions. Furthermore, the reliance on cross-sectional data in 21 of the 23 studies means that it is not possible to disentangle cause and effect, and longer-term outcomes of the error are neglected. The latter may be key in the management of error. The links drawn between the severity of an error and the emotional response are questionable because of the use of selfreports to measure severity. ${ }^{4} 1926$ A final concern is that although there is diversity across the studies in terms of job types, specialism and socialisation into medicine, only one study uses a large multidisciplinary sample. ${ }^{27}$ Many other studies focus specifically on one group, for example physicians, nurses and medical trainees, and are often reliant on small samples. It is therefore difficult to draw conclusions across study findings, and to generalise beyond each sampled group.

\section{Methodological weaknesses of review}

First, it is possible that the search strategy did not locate all the relevant studies. However, this possibility was minimised by 
consulting an information scientist in the development of search terms and the selection of databases. Nonetheless, even in the event of a faultless search strategy, the problem of publication bias means that important negative findings from unpublished research may have been omitted.

Furthermore, there is evidence that the level of sensitivity and precision of bibliographic databases is dependent on the topic searched, and this may affect the number of articles returned. ${ }^{38}$ The efficacy of database searches to capture articles in the area of patient safety appears yet to be evaluated, but the implication from other health-related research is that some articles will be missing. ${ }^{38} 39$ This issue was addressed through the use of several databases to broaden coverage, and additional hand searching.

\section{SUMMARY}

This review identified 23 studies investigating the response and coping of health professionals following experience of an error. Consistent findings support the existence of an intense emotional response, and the impact of error on individuals' professional and personal lives. Current research fails to fully address how individuals cope with making an error, and the longer-term impact of this on themselves and those around them, including their patients. Barriers that prevent professionals receiving the necessary support include the negative attitudes towards error in the culture of medicine, the threat of professional loss and the lack of available institutional support. Further research requires the exploration of the full life cycle of clinician experiences following error, from immediate responses to longer-term impact, as well as the role of organisational culture and preregistration education.

Funding Bradford Institute for Health Research, Temple Bank House, Duckworth Lane, Bradford BD9 6RJ.

\section{Competing interests None.}

Provenance and peer review Not commissioned; externally peer reviewed.

\section{REFERENCES}

1. Wears RL, Wu AW. Dealing with failure: the aftermath of errors and adverse events. Ann Emerg Med 2000;39:344-6.

2. Schwappach DLB, Boluarte TA. The emotional impact of medical error involvement on physicians: a call for leadership and organisational accountability. Swiss Med Wkly 2009;139:9-15.

3. White AA, Waterman AD, McCotter $P$, et al. Supporting health care workers after medical error: considerations for health care leaders. J Clin Outcomes Manag 2008:15:240-7.

4. Muller D, Ornstein K. Perceptions of and attitudes towards medical errors among medical trainees. Med Educ 2007;41:645-52.

5. Harden A, Thomas J. Methodological issue in combining diverse study types in systematic reviews. Int J Soc Res Methodol 2005;8:257-71.

6. Peat M, Entwhistle V, Hall J, et al. A scoping review and approach to appraisal of interventions intended to involve patients in patient safety. $J$ Health Serv Res Policy 2010;15:17-25.

7. Jackson C, Cheater FM, Reid I. A systematic review of decision support needs of parents making child health decisions. Health Expect 2008;11:232-51.

8. Thomas $\mathbf{J}$, Harden A. Methods for the thematic synthesis of qualitative research in systematic reviews. BMC Med Res Methodol 2008;8:45

9. Edwards A, Hood K, Matthews E, et al. The effectiveness of one-to-one riskcommunication interventions in health care: a systematic review. Med Decis Making 2000;20:290-7.
10. Thomas J, Sutcliffe K, Harden A. Children and healthy eating: a systematic review of barriers and facilitators. London: EPPI Centre, Social Science Research Unit, Institute of Education, University of London, 2003.

11. Landis JR, Koch GG. Measurement of observer agreement for categorical data. Biometrics 1977;33:159-74.

12. Hughes EC. Mistakes at work. Canadian J Econ Polit Sci 1951;17:320-7.

13. Waterman AD, Garbutt J, Hazel E, et al. The emotional impact of medical errors on practicing physicians in the United States and Canada. Jt Comm J Qual Patient Saf 2007:33:467-76

14. Schelbred AB, Nord R. Nurses' experiences of drug administration errors. J Adv Nurs 2007;60:317-24.

15. Fisseni G, Pentzek M, Abholz $\mathrm{H}$. Responding to serious medical error in general practice consequences for the GPs involved: analysis of 75 cases from Germany. Fam Pract 2007:71:9-13.

16. Kroll L, Singleton A, Collier J, et al. Learning not to take it seriously: junior doctors accounts of error. Med Educ 2008;42:982-90.

17. Scott SD, Hirschinger LE, Cox KR, et al. The natural history of recovery for the healthcare provider 'second victim' after adverse patient events. Qual Saf Health Care 2009;18:325-30.

18. Mizrahi T. Managing medical mistakes: ideology, insularity and accountability among internists-in-training. Soc Sci Med 1984;19:135-46.

19. Engel KG, Rosenthal M, Sutcliffe KM. Residents' responses to medical error: coping learning, and change. Acad Med 2006:81:86-93.

20. Hobgood C, Hevia A, Tamayo-Sarver JH, et al. The influence of the causes and contexts of medical errors on emergency medicine residents' responses to their errors: an exploration. Acad Med 2005:80:758-64.

21. West CP, Huschka MM, Novotny PJ, et al. Association of perceived medical errors with resident distress and empathy - a prospective longitudinal study. JAMA 2006;296:1071-8.

22. Cunningham $\mathbf{W}$. The immediate and long-term impact on New Zealand doctors who receive patient complaints. N Z Med J 2004;117:972

23. Lander LI, Connor JA, Shah RK, et al. Otolaryngologists' responses to errors and adverse events. Laryngoscope 2006;116:1114-20.

24. Arndt M. Nurses' medication errors. J Adv Nurs 1994:19:519-26.

25. Kaldjian LC, Jones EW, Wu BJ, et al. Reporting medical errors to improve patien safety-Survey of physicians in teaching hospitals. Arch Intern Med 2008:168:40-6.

26. Meurier CE, Vincent CA, Parmar DG. Nurses' responses to severity dependent errors: a study of the causal attributions made by nurses following an error. $J$ Adv Nurs 1998;27:349-54.

27. Wolf ZR, Serembus J, Smetzer J, et al. Responses and concerns of healthcare providers to medication errors. Clin Nurse Spec 2000;14:278-90.

28. Christensen JF, Levinson W, Dunn PM. The heart of darkness-the impact of perceived mistakes on physicians. J Gen Intern Med 1992; 7:424-31.

29. Wu AW, Folkman S, McPhee SJ, et al. Do house officers learn from their mistakes? Qual Saf Health Care 2003;12:221-7.

30. Crigger NJ, Meek VL. Toward a theory of self-reconciliation following mistakes in nursing practice. J Nurs Scholarsh 2007;39:177-83.

31. Martinez W, Lo B. Medical students' experiences with medical errors: an analysis of medical student essays. Med Educ 2008:42:733-41.

32. Aasland 0G, Forde R. Impact of feeling responsible for adverse events on doctors personal and professional lives: the importance of being open to criticism from colleagues. Oual Saf Health Care 2005:14:13-17.

33. Gallagher TH, Waterman AD, Ebers AG, et al. Patients' physicians' attitudes regarding the disclosure of medical errors. JAMA 2003:289:1001-7.

34. Fischer MA, Mazor KM, Baril J, et al. Learning from mistakes-factors that influence how students and residents learn from medical errors. J Gen Intern Med 2006;21:419-23.

35. Newman MC. The emotional impact of mistakes on family physicians. Arch Fam Med 1996;5:71-5.

36. Goldberg DP, Williams P. A user's guide to the general health questionnaire. Berkshire, England: Nelson, 1988.

37. Beck AT, Ward $\mathrm{CH}$, Mendelson $\mathrm{M}$, et al. An inventory for measuring depression. Arch Gen Psychiatry 1961:4:561-71.

38. Watson RJD, Richardson PH. Accessing the literature on outcome studies in group psychotherapy: the sensitivity and precision of Medline and PsycINFO bibliographic database searching. Br J Med Psychol 1999:72:127-34.

39. Mcdonald S, Taylor L, Adams C. Searching the right database. A comparison of four databases for psychiatry journals. Health Libr Rev 1999:16:151-6. 